Provided for non-commercial research and education use. Not for reproduction, distribution or commercial use.

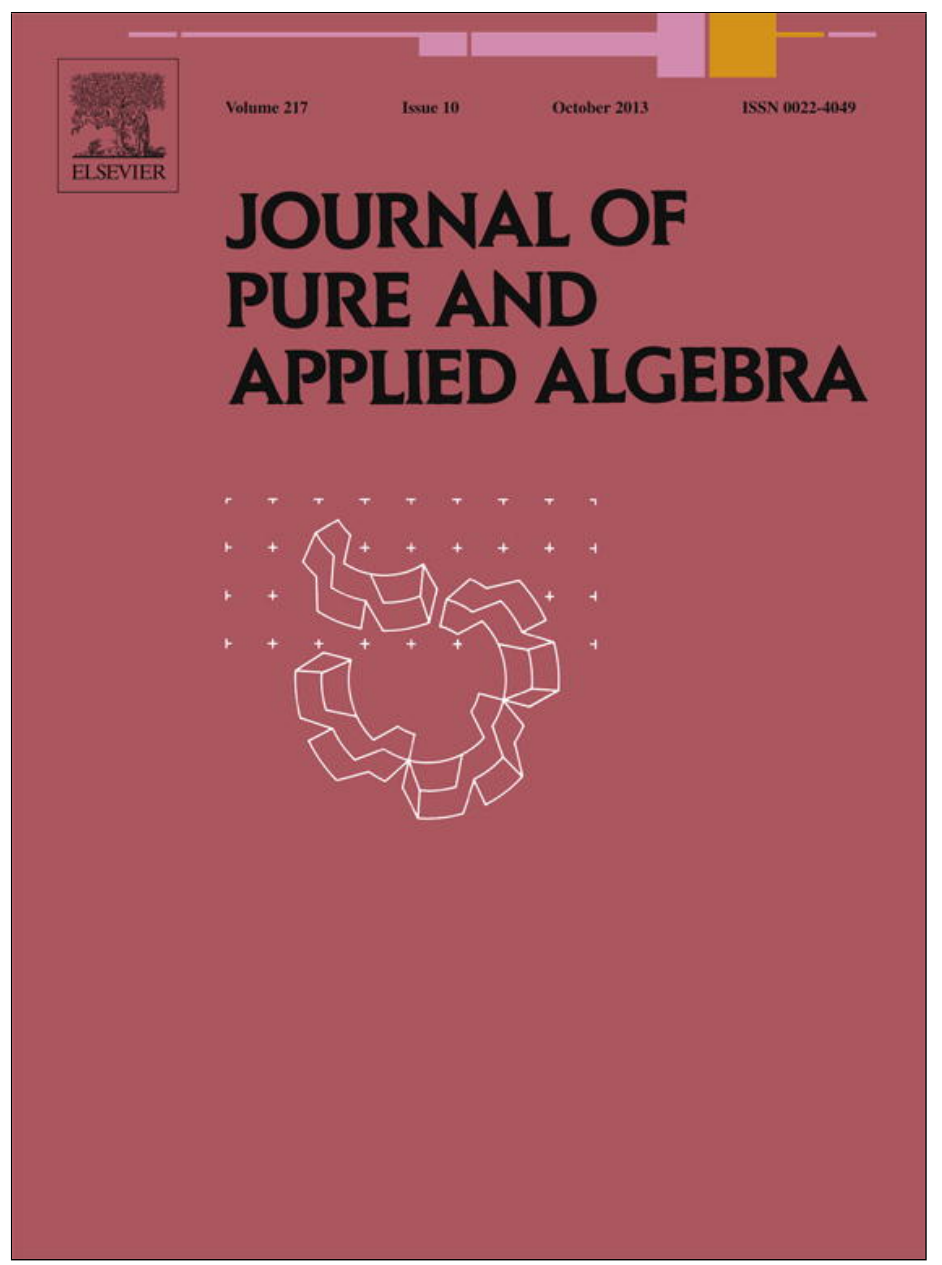

This article appeared in a journal published by Elsevier. The attached copy is furnished to the author for internal non-commercial research and education use, including for instruction at the authors institution and sharing with colleagues.

Other uses, including reproduction and distribution, or selling or licensing copies, or posting to personal, institutional or third party websites are prohibited.

In most cases authors are permitted to post their version of the article (e.g. in Word or Tex form) to their personal website or institutional repository. Authors requiring further information regarding Elsevier's archiving and manuscript policies are encouraged to visit:

http://www.elsevier.com/authorsrights 


\section{Brill-Noether with ramification at unassigned points}

\section{Gavril Farkas}

Humboldt-Universität zu Berlin, Institut Für Mathematik, Unter den Linden 6, 10099 Berlin, Germany

\section{A R T I C L E I N F O}

\section{Article history:}

Received 19 July 2012

Received in revised form 21 January 2013

Available online 15 February 2013

Communicated by R. Vakil

\section{A B S T R A C T}

We discuss, how via limit linear series and standard facts about divisors on moduli spaces of pointed curves, one can establish a non-existence Brill-Noether results for linear series with prescribed ramification at unassigned points.

(c) 2013 Elsevier B.V. All rights reserved.

MSC: $14 \mathrm{H} 10 ; 14 \mathrm{H} 51$

In the course of developing their theory of limit linear series, among many other applications, Eisenbud and Harris [2,3] also considered the Brill-Noether problem with prescribed ramification at assigned points. For a smooth curve $C$ of genus $g$, a point $p \in C$ and a linear series $\ell=(L, V) \in G_{d}^{r}(C)$, one denotes by

$$
\alpha^{\ell}(p): 0 \leq \alpha_{0}^{\ell}(p) \leq \cdots \leq \alpha_{r}^{\ell}(p) \leq d-r
$$

the ramification sequence of $\ell$ at $p$.

Having fixed points $p_{1}, \ldots, p_{n} \in C$, Schubert indices $\bar{\alpha}^{j}: 0 \leq \alpha_{0}^{j} \leq \cdots \leq \alpha_{r}^{j} \leq d-r$ of type $(r, d)$ for $j=1, \ldots, n$, the locus of linear series on $C$ having prescribed ramification at $p_{1}, \ldots, p_{n} \in C$, that is,

$$
G_{d}^{r}\left(C,\left(p_{j}, \bar{\alpha}^{j}\right)\right):=\left\{\ell \in G_{d}^{r}(C): \alpha^{\ell}\left(p_{j}\right) \geq \bar{\alpha}^{j} \text { for } j=1, \ldots, n\right\}
$$

is a generalized determinantal variety of expected dimension

$$
\rho\left(g, r, d, \bar{\alpha}^{1}, \ldots, \bar{\alpha}^{n}\right):=\rho(g, r, d)-\sum_{j=1}^{n} \sum_{i=0}^{r} \alpha_{i}^{j},
$$

where $\rho(g, r, d):=g-(r+1)(g-d+r)$ is the Brill-Noether number. It is proved in [3] Theorem 1.1, that for a general pointed curve $\left[C, p_{1}, \ldots, p_{n}\right] \in \mathcal{M}_{g, n}$, each component of $G_{d}^{r}\left(C,\left(p_{j}, \bar{\alpha}^{j}\right)\right)$ has dimension $\rho\left(g, r, d, \bar{\alpha}^{1}, \ldots, \bar{\alpha}^{n}\right)$. For $n=1$, a necessary and sufficient condition for existence is given. Denoting the positive part of an integer $n \in \mathbb{Z}$ by $(n)_{+}:=\max \{n, 0\}$, the pointed curve $[C, p] \in \mathcal{M}_{g, 1}$ carries a linear series $\ell \in G_{d}^{r}(C)$ with ramification $\alpha^{\ell}(p) \geq \bar{\alpha}$ if and only if

$$
\sum_{i=0}^{r}\left(\alpha_{i}+g-d+r\right)_{+} \leq g
$$

Setting $\bar{\alpha}=(0, \ldots, 0)$, one recovers the Brill-Noether theorem. In this note, we explain how the methods of [3,4] coupled with basic facts about $\operatorname{Pic}\left(\overline{\mathcal{M}}_{g, n}\right)$ provide a solution to the Brill-Noether problem with prescribed ramification at unassigned points.

E-mail address: farkas@math.hu-berlin.de. 
Theorem 0.1. Let $C$ be a general curve of genus $g \geq 2$ and $\bar{\alpha}: 0 \leq \alpha_{0} \leq \cdots \leq \alpha_{r} \leq d-r$ a Schubert ramification index. If

$$
\rho(g, r, d)-\sum_{i=0}^{r} \alpha_{i}<-1
$$

then $C$ carries no linear series $\ell \in G_{d}^{r}(C)$ having ramification $\alpha^{\ell}(p) \geq \bar{\alpha}$ at some point $p \in C$.

As expected, since on an elliptic curve, due to the existence of translations, one cannot speak of an unassigned point, Theorem 0.1 is true for every smooth curve of genus 1 . The inequality $\rho\left(1, r, d, \alpha^{\ell}(p)\right) \geq 0$ holds for every elliptic curve $[E, p] \in \mathcal{M}_{1,1}$ and every linear series $\ell \in G_{d}^{r}(E)$, and lies at the heart of the proof in [2] of the non-existence part of the Brill-Noether theorem by degeneration to a flag curve with $g$ elliptic tails. We note that, some partial results in the direction of Theorem 0.1 were obtained in [6]. The previous result can be, to some extent, generalized to multiple points:

Theorem 0.2. We fix integers $r, d \geq 1$ and $g \geq 2$ as well as Schubert indices $\bar{\alpha}^{1}, \ldots, \bar{\alpha}^{n}$, with

$$
\rho\left(g, r, d, \bar{\alpha}^{1}, \ldots, \bar{\alpha}^{n}\right)<-1 .
$$

Then the locus $\left\{\left[C, p_{1}, \ldots, p_{n}\right] \in \mathcal{M}_{g, n}: G_{d}^{r}\left(C,\left(p_{j}, \bar{\alpha}^{j}\right)\right) \neq \emptyset\right\}$ has codimension at least two in moduli.

Setting $n=1$, one recovers Theorem 0.1. Contrary to our first result, Theorem 0.2 is not true in genus 1 and fails already on $\mathcal{M}_{1,2}$. Indeed, for an integer $a \geq 2$, we fix an elliptic curve $E$ and distinct points $p_{1}, p_{2} \in E$ such that $p_{1}-p_{2} \in \operatorname{Pic}^{0}(E)[a]$. Choose a rational function $f \in \mathbb{C}(E)$ with $\operatorname{div}(f)=a \cdot p_{2}-a \cdot p_{1}$ and consider the linear series $\ell:=\left(\mathcal{O}_{E}\left(2 a \cdot p_{1}\right), V\right) \in G_{2 a}^{2}(E)$, where $V:=\left\langle 1, f, f^{2}\right\rangle$. Clearly $\alpha^{\ell}\left(p_{j}\right)=(0, a-1,2 a-2)$ for $j=1,2$, hence one calculates $\rho\left(1,2,2 a, \alpha^{\ell}\left(p_{1}\right), \alpha^{\ell}\left(p_{2}\right)\right)=-2$. On the other hand, the locus $D_{a}:=\left\{\left[E, p_{1}, p_{2}\right] \in \mathcal{M}_{1,2}: p_{1}-p_{2} \in \operatorname{Pic}^{0}(E)[a]\right\}$ is obviously a divisor in the moduli space. Considering higher dimensional linear series, the same idea produces counterexamples with even more negative Brill-Noether number.

Straightforward generalizations of Theorem 0.2 to lower Brill-Noether numbers and higher codimension in moduli are false when $r \geq 2$, as the following example illustrates. Let $C$ be a curve of genus 2 and $L=K_{C}^{\otimes 2} \in G_{4}^{2}(C)$. If $p \in C$ is a Weierstrass point, then $\alpha^{L}(p)=(0,1,2)$. In particular, if both $p_{1}$ and $p_{2}$ are Weierstrass points, then we obtain that $\rho\left(2,2,4, \alpha^{L}\left(p_{1}\right), \alpha^{L}\left(p_{2}\right)\right)=-4$. On the other hand, the codimension of the locus

$$
\left\{\left[C, p_{1}, p_{2}\right] \in \mathcal{M}_{2,2}: G_{4}^{2}\left(C,\left(p_{j},(0,1,2)\right)\right) \neq \emptyset\right\}
$$

is two, in particular it projects onto $\mathcal{M}_{2}$. Similar examples can be constructed for arbitrary genus and, in this sense, Theorem 0.2 is optimal. Even though these counterexamples invite caution before formulating new predictions, we believe the following statement should be true:

Conjecture 0.3. Let $g, r, d$ be positive integers and $\bar{\alpha}$ a Schubert index of type $(r, d)$ with $\rho(g, r, d, \bar{\alpha})<-2$. Then the locus of curves $[C] \in \mathcal{M}_{g}$ carrying a linear series $\ell \in G_{d}^{r}(C)$ with $\alpha^{\ell}(p) \geq \bar{\alpha}$ for some point $p \in C$, has codimension at least two in moduli.

\section{The proofs}

The proof of Theorem 0.1 is an adaptation of an idea that appears in [4]. First, by explicit methods, we prove Theorem 0.1 in genus 3. Then, assuming by contradiction that a general curve $[C] \in \mathcal{M}_{g}$ possesses a point $p \in C$ with $G_{d}^{r}(C,(p, \bar{\alpha})) \neq \emptyset$, the locus of such pairs $[C, p]$ in the universal curve $\mathcal{M}_{g, 1}$ is an effective divisor. By using the solution to the problem in genus 3 and basic facts about the Picard group of $\overline{\mathcal{M}}_{g, 1}$ (that have already been used in [3,4]), we show that this effective divisor must be empty, thus establishing Theorem 0.1 . Theorem 0.2 is proved by induction and relies on the already established case $n=1$.

We work throughout over the complex numbers and we assume familiarity with the theory of limit linear series [2]. We begin with some preliminaries on moduli spaces of curves and recall that for $g \geq 3$ and $n \geq 1$, the rational Picard group $\operatorname{Pic}\left(\overline{\mathcal{M}}_{g, n}\right)$ is freely generated by the Hodge class $\lambda$, the relative cotangent classes $\psi_{1}, \ldots, \bar{\psi}_{n}$, the boundary divisor class $\delta_{\text {irr }}:=\left[\Delta_{\text {irr }}\right]$ of irreducible $n$-pointed stable curves of curves $g$ and by the classes $\delta_{i: S}:=\left[\Delta_{i: S}\right]$, where for each $i \geq 0$ and $S \subset\{1, \ldots, n\}$, the general point of the boundary divisor $\Delta_{i: S}$ corresponds to a transverse union of two smooth curves of genus $i$ and $g-i$ respectively, meeting in one point, the marked points lying on the genus $i$ component being precisely those labeled by $S$. Obviously $\delta_{i: S}=\delta_{g-i: S^{c}}$. When $n=0$, we write as usually $\delta_{i}:=\delta_{i: \emptyset} \in \operatorname{Pic}\left(\overline{\mathcal{M}}_{g}\right)$ for $i=0, \ldots,\left\lfloor\frac{g}{2}\right\rfloor$. Similarly, for $n=1$, we write $\delta_{i}:=\delta_{i:\{1\}} \in \operatorname{Pic}\left(\overline{\mathcal{M}}_{g, 1}\right)$, for $i=1, \ldots, g-1$. The following well-known fact will be used in the course of the proof of Theorem 0.2 :

Lemma 1.1. Let $g \geq 3$ and $\mathscr{D}$ an effective divisor on $\mathcal{M}_{g, n}$ such that all the $\lambda, \psi_{1}, \ldots, \psi_{n}$ and $\left\{\delta_{0: S}\right\}_{|S|=2}$-coefficients in the expansion of the class $[\bar{D}] \in \operatorname{Pic}\left(\overline{\mathcal{M}}_{g, n}\right)$ of its closure in $\overline{\mathcal{M}}_{g, n}$ are equal to zero. Then $\mathscr{D}=0$. 
Proof. Let $\pi: \overline{\mathcal{M}}_{g, n} \rightarrow \overline{\mathcal{M}}_{g}$ be the forgetful morphism. If $\pi(\bar{D})=\overline{\mathcal{M}}_{g}$, then there exists an index $1 \leq i \leq n$ such that $\bar{D}$ intersects non-trivially the general fiber $F_{i}$ of the morphism $\pi_{i}: \overline{\mathcal{M}}_{g, n} \rightarrow \overline{\mathcal{M}}_{g, n-1}$ forgetting the $i$-th marked point. Note that, $F_{i} \cdot \psi_{i}=2 g-3+n$, as well as $F_{i} \cdot \psi_{j}=1$ and $F_{i} \cdot \delta_{0: j i}=1$ for $j \in\{i\}^{c}$, whereas the intersection numbers with all other generators of $\operatorname{Pic}\left(\overline{\mathcal{M}}_{g, n}\right)$ are zero. We obtain that, $F_{i} \cdot \bar{D}=0$, a contradiction. Therefore, $\mathscr{D}=\pi^{*}\left(\mathscr{D}^{\prime}\right)$, where $\mathscr{D}^{\prime}$ is an effective divisor on $\mathcal{M}_{g}$. Since the Hodge class on $\overline{\mathcal{M}}_{g, n}$ is pulled back via $\pi$ via the Hodge class on $\overline{\mathcal{M}}_{g}$ and the map $\pi^{*}: \operatorname{Pic}\left(\overline{\mathcal{M}}_{g}\right) \rightarrow \operatorname{Pic}\left(\overline{\mathcal{M}}_{g, n}\right)$ is injective, it follows that the $\lambda$-coefficient in the expansion of $\left[\overline{\mathcal{D}}^{\prime}\right] \in \operatorname{Pic}\left(\overline{\mathcal{M}}_{g}\right)$ is equal to 0 as well. But $\mathcal{M}_{g}$ admits a compactification, namely the Satake compactification $\overline{\mathcal{M}}_{g}^{s}$, having boundary $\overline{\mathcal{M}}_{g}^{s}-\mathcal{M}_{g}$ of codimension 2; in fact, there is a regular Torelli map $t: \overline{\mathcal{M}}_{g} \rightarrow \overline{\mathcal{M}}_{g}^{S}$, assigning to a stable curve [C] $\in \overline{\mathcal{M}}_{g}$ the product of the degree zero Jacobian varieties of the components of its normalization $\widetilde{C}$ of $C$, and under this map $t_{*}\left(\Delta_{i}\right)=0$, for $i=0, \ldots,\left\lfloor\frac{g}{2}\right\rfloor$. A consequence of the existence of $\overline{\mathcal{M}}_{g}^{s}$ is that there exists no effective divisor $\mathscr{D}^{\prime}$ on $\mathcal{M}_{g}$ whose $\lambda$-coefficient is zero, hence $\mathscr{D}^{\prime}=0$, and thus $\mathscr{D}=0$.

Following [3], we consider the clutching map $\varphi_{g, n}: \overline{\mathcal{M}}_{0, g+n} \rightarrow \overline{\mathcal{M}}_{g, n}$, given by

$$
\varphi_{g, n}\left(\left[R, x_{1}, \ldots, x_{g}, p_{1}, \ldots, p_{n}\right]\right):=\left[R \cup_{x_{1}} E_{1} \cup \cdots \cup_{x_{g}} E_{g}, p_{1}, \ldots, p_{n}\right],
$$

obtained by gluing fixed elliptic curves $E_{1}, \ldots, E_{g}$ at the first $g$ marked points of a curve $R$ of arithmetic genus 0 . The marked points $p_{1}, \ldots, p_{n}$ of the resulting genus $g$ stable curve lie on the rational spine $R$. We consider the action of the symmetric group $\mathfrak{S}_{g}$ on $\overline{\mathcal{M}}_{0, g+n}$ by permuting the marked points labeled by $x_{1}, \ldots, x_{g}$. For a subset $S \subset\left\{p_{1}, \ldots, p_{n}\right\}$ and an integer $0 \leq i \leq g$ with $2 \leq|S|+i \leq g+n-2$, we define the $\mathfrak{S}_{g}$-invariant boundary divisor

$$
B_{i}^{S}:=\sum_{\substack{T \subset\left\{x_{1}, \ldots, x_{g}\right\} \\|T|=i}} \delta_{0: S \cup T} \in \operatorname{Pic}\left(\overline{\mathcal{M}}_{0, g+n}\right)^{\mathcal{S}_{g}} .
$$

Clearly $\varphi_{g, n}^{*}\left(\delta_{i: S}\right)=B_{i}^{S}$, whenever $i+|S| \geq 2$, whereas $\varphi_{g, n}^{*}\left(\psi_{i}\right)=\psi_{p_{i}}$. In order to distinguish between cotangent classes on $\overline{\mathcal{M}}_{0, g+n}$ and $\overline{\mathcal{M}}_{g, n}$, we label the corresponding marked point by $p_{i}$ on the genus zero curve and by $i \in\{1, \ldots, n\}$ on the genus $g$ curve.

Lemma 1.2. The $\mathfrak{S}_{g}$-invariant rational Picard group Pic $\left(\overline{\mathcal{M}}_{0, g+2}\right)^{\mathfrak{S}_{g}}$ is freely generated by the boundary classes $\left\{B_{i}^{p_{1}}\right\}_{i=1}^{g-1}$ and $\left\{B_{j}^{p_{1} p_{2}}\right\}_{j=1}^{g-2}$. In particular, $\operatorname{dim}_{\mathbb{Q}} \operatorname{Pic}\left(\overline{\mathcal{M}}_{0, g+2}\right)^{\mathfrak{S}_{g}}=2 g-3$.

Proof. This follows for instance from [5] Proposition 1. It is shown in loc. cit. that, there is precisely one relation between the $\mathfrak{S}_{g}$-invariant boundary divisor classes, which expresses $B_{0}^{p_{1} p_{2}}=\delta_{0: p_{1} p_{2}}$ in terms of all the other invariant boundary classes. The cotangent classes are also expressible in this basis, see [5] Lemma 1:

$$
\psi_{p_{1}}=\sum_{j=1}^{g-1} \frac{(g+1-j)(g-j)}{(g+1) g}\left(B_{j}^{p_{1}}+B_{j-1}^{p_{1} p_{2}}\right) .
$$

We fix a general pointed curve $\left[C, p^{\prime}, q\right] \in \mathcal{M}_{g-3,2}$ and consider another clutching map

$$
j: \overline{\mathcal{M}}_{3,1} \rightarrow \overline{\mathcal{M}}_{g, 1}, j([B, p]):=\left[B \cup_{p \sim p^{\prime}} C, q\right] .
$$

The following formulas are easy to prove, see for instance the proof of Lemma 4.3 in [4] or Lemma 3.3 in [1]:

$$
j^{*}(\lambda)=\lambda, \quad j^{*}(\psi)=0, \quad j^{*}\left(\delta_{0}\right)=0, \quad j^{*}\left(\delta_{g-3}\right)=-\psi, \quad j^{*}\left(\delta_{g-2}\right)=\delta_{1}, \quad j^{*}\left(\delta_{g-1}\right)=\delta_{2},
$$

and $j^{*}\left(\delta_{i}\right)=0$, for $i=1, \ldots, g-4$.

The next result establishes Theorem 0.1 for $g=3$. In genus 2, it is shown in [4] Lemma 3.3, that the inequality $\rho\left(2, r, d, \alpha^{\ell}(p)\right) \geq-1$ holds for any pointed curve $[C, p] \in \mathcal{M}_{2,1}$ and any linear series $\ell \in G_{d}^{r}(C)$, with equality if and only if $p \in C$ is a Weierstrass point and $\ell=|(r+2) \cdot p|+(d-r-2) \cdot p$. This establishes Theorem 0.1 in genus 2 . The genus 3 analogue of this statement will be the starting step in our induction argument.

Proposition 1.3. Let $[C, p] \in \mathcal{M}_{3,1}$ be a pointed curve of genus 3 and $\ell \in G_{d}^{r}(C)$. If $\rho\left(3, r, d, \alpha^{\ell}(p)\right) \leq-2$, then either $p \in C$ is a hyperflex, that is, $K_{C}=\mathcal{O}_{C}(4 p)$, or else, $C$ is hyperelliptic and $p \in C$ is a Weierstrass point. In particular, for any Schubert index $\bar{\alpha}: \alpha_{0} \leq \cdots \leq \alpha_{r} \leq d-r$ such that $\rho(3, r, d, \bar{\alpha}) \leq-2$, each component of the locus

$$
\left\{[C, p] \in \mathcal{M}_{3,1}: G_{d}^{r}(C,(p, \bar{\alpha})) \neq \emptyset\right\}
$$

has codimension at least 2.

Proof. From Riemann-Roch it follows that if $\Lambda \in G_{d}^{r}(C)$ is a linear series with $r \geq 1$, then the inequality $d \geq 3+r$ always holds except when $r=2$ and $\Lambda=\left|K_{C}\right|$, or when $r=1$. Then $d=3$ and $\Lambda=\left|K_{C}(-p)\right| \in W_{3}^{1}(C)$ when $C$ is non-hyperelliptic, or $d \in\{2,3\}$ when $C$ is hyperelliptic respectively. 
We fix now, a linear series $\ell \in G_{d}^{r}(C)$ and let $a_{i}:=a_{i}^{\ell}(p)=\alpha_{i}^{\ell}(p)+i$ be the $i$-th entry in the vanishing sequence of $\ell$ at $p$. By definition, then $\ell\left(-a_{i} \cdot p\right) \in G_{d-a_{i}}^{r-i}(C)$, for each $i=0, \ldots, r$. Assume first that $C$ is non-hyperelliptic. Applying the previous observation, we write the following inequalities:

$$
\begin{array}{ll}
d-a_{r-i} \geq i+3 & \text { for } i=0, \ldots, r-3, \quad \text { and } \\
d-a_{r-2} \geq 4, \quad d-a_{r-1} \geq 3, \quad d-a_{r} \geq 0 .
\end{array}
$$

Adding these inequalities up, we obtain that $(r+1)(d-r)-\sum_{i=0}^{r} \alpha_{i}^{\ell}(p) \geq 3 r-2$, or equivalently, $\rho\left(3, r, d, \alpha^{\ell}(p)\right) \geq-2$. Equality holds, only if the three inequalities appearing in (3) are all equalities. When this happens, then $\left(a_{r-2}, a_{r-1}, a_{r}\right)=$ $(d-4, d-3, d)$ and we find that $h^{0}\left(C, \mathcal{O}_{C}(4 p)\right)=3$, that is, $p \in C$ is a hyperflex. This condition defines a codimension 2 subvariety of $\overline{\mathcal{M}}_{3,1}$. Thus, when $p \in C$ is not a hyperflex, then $\rho\left(g, r, d, \alpha^{\ell}(p)\right) \geq-1$.

Assume now that $C$ is hyperelliptic. The inequalities in (2) still hold, as well as, $d-a_{r-2} \geq 4$ and $d-a_{r} \geq 0$. Assuming that $a_{r-1}=2$, one finds that $h^{0}\left(C, \mathcal{O}_{C}(2 p)\right)=2$, that is, $p \in C$ is a Weierstrass point.

Proof of Theorem 0.1. We fix a Schubert index $\bar{\alpha}: \alpha_{0} \leq \cdots \leq \alpha_{i} \leq d-r$, such that $\rho(g, r, d, \bar{\alpha})<-1$, and assume that for a general curve $C$, there exists a point $p \in C$ with $G_{d}^{r}(C, p, \bar{\alpha}) \neq \emptyset$. The locus $\mathscr{D}:=\left\{[C, p] \in \mathcal{M}_{g, 1}: G_{d}^{r}(C, p, \bar{\alpha}) \neq \emptyset\right\}$ is then a divisor in $\mathcal{M}_{g, 1}$ (by using the result of Eisenbud and Harris [3] mentioned in the introduction, the possibility $\mathscr{D}=\mathcal{M}_{g, 1}$ can be ruled out). We express the class of its closure $\bar{D}$ in $\overline{\mathcal{M}}_{g, 1}$ in terms of the generators of $\operatorname{Pic}\left(\overline{\mathcal{M}}_{g, 1}\right)$ :

$$
[\bar{D}]=a \lambda+c \psi-b_{\text {irr }} \delta_{\text {irr }}-\sum_{i=1}^{g-1} b_{i} \delta_{i} .
$$

A flag curve $X$ having a rational spine consisting of a tree of $\mathbf{P}^{1}$ s and $g$ elliptic tails satisfies the pointed Brill-Noether theorem with respect to marked points lying on the rational spine, see [3] Theorem 1.1; precisely, if $p_{1}, \ldots, p_{n}$ are smooth points of $X$ not lying on any of the elliptic tails, then $\rho\left(g, r, d, \alpha^{\ell}\left(p_{1}\right), \ldots, \alpha^{\ell}\left(p_{n}\right)\right) \geq 0$, for any limit linear series $\ell$ of type $\mathfrak{g}_{d}^{r}$ on $X$. It thus follows that, $\operatorname{Im}\left(\varphi_{g, 1}\right) \cap \bar{D}=\emptyset$, in particular $\varphi_{g, 1}^{*}([\bar{D}])=0$. Using the description in [4] Lemma 4.2 for the pull-back map at the level of divisors $\varphi_{g, 1}^{*}: \operatorname{Pic}\left(\overline{\mathcal{M}}_{g, 1}\right) \rightarrow \operatorname{Pic}\left(\overline{\mathcal{M}}_{0, g+1}\right)$, we obtain that for $i=1, \ldots, g-2$ the following relations hold:

$$
b_{i}=\frac{(g-i)(g-i-1)}{g(g-1)} c+\frac{i(g-i)}{g-1} b_{g-1} .
$$

Next we employ Proposition 1.3. From the additivity of the Brill-Noether number, we find that the pointed curve $\left[B \cup_{p} C, q\right] \in \overline{\mathcal{M}}_{g, 1}$ carries no limit linear series $\ell \in G_{d}^{r}(C)$ with $\rho\left(g, r, d, \alpha^{\ell}(q)\right)<-1$, therefore, $\left[j^{*}(\bar{D})\right]=0$, where we recall that $j: \overline{\mathcal{M}}_{3,1} \rightarrow \overline{\mathcal{M}}_{g, 1}$ was the clutching map attaching a fixed pointed curve of genus $g-3$. In terms of coefficients of $[\overline{\mathcal{D}}]$ this information is expressed as follows:

$$
0=\left[j^{*}(\bar{D})\right]=a \lambda-b_{\text {irr }} \delta_{\text {irr }}+b_{g-3} \psi-b_{g-2} \delta_{1}-b_{g-1} \delta_{2} \in \operatorname{Pic}\left(\overline{\mathcal{M}}_{3,1}\right) .
$$

From the independence of the boundary divisor classes on $\overline{\mathcal{M}}_{3,1}$, we find that $a=b_{\text {irr }}=b_{g-1}=b_{g-2}=b_{g-3}=0$. Using repeatedly (4), we find that $[\overline{\mathscr{D}}]=0 \in \operatorname{Pic}\left(\overline{\mathcal{M}}_{g, 1}\right)$, that is, $\mathscr{D}=0$. The contradiction comes from the assumption that $\mathscr{D}$ was of codimension one in $\mathcal{M}_{\mathrm{g}, 1}$.

Remark 1.4. The argument above is quite close to that in [4], where instead of the map $j$, the clutching map $\iota: \overline{\mathcal{M}}_{2,1} \rightarrow \overline{\mathcal{M}}_{g, 1}$ obtained by attaching a fixed 2-pointed curve of genus $g-2$ at the marked point of each stable curve of genus 2 is considered. It is showed in [4] Lemma 4.3 that $\left[\iota^{*}(\bar{D})\right]=0$. However, this does not appear to be enough in order to conclude that $[\bar{D}]=0$ and finish the proof. Indeed, in view of Mumford's relation $10 \lambda=\delta_{\text {irr }}+2 \delta_{1}$ on $\overline{\mathcal{M}}_{2,1}$, there exists a one-dimensional vector space of divisor classes $\gamma \in \operatorname{Pic}\left(\overline{\mathcal{M}}_{g, 1}\right)$ satisfying both constraints $\varphi^{*}(\gamma)=0$ and $\iota^{*}(\gamma)=0$. To circumvent this problem, we considered instead the map $j: \overline{\mathcal{M}}_{3,1} \rightarrow \overline{\mathcal{M}}_{g, 1}$. This also explains why the induction in the proof of Theorem 0.1 starts only from genus 3.

We split the proof of Theorem 0.2 in two parts.

Proof of Theorem 0.2 (the case $n=2$ ). Let $\bar{\alpha}^{1}$ and $\bar{\alpha}^{2}$ be Schubert indices of type $(r, d)$ such that $\rho\left(g, r, d, \bar{\alpha}^{1}, \bar{\alpha}^{2}\right)<-1$ and assume by contradiction that the locus

$$
D:=\left\{\left[C, p_{1}, p_{2}\right] \in \mathcal{M}_{g, 2}: G_{d}^{r}\left(C,\left(p_{1}, \bar{\alpha}^{1}\right),\left(p_{2}, \bar{\alpha}^{2}\right)\right) \neq \emptyset\right\},
$$

is an effective divisor. We express the class of its closure in $\overline{\mathcal{M}}_{g, 2}$ as a combination

$$
[\bar{D}]=a \lambda+c_{1} \psi_{1}+c_{2} \psi_{2}-b_{\mathrm{irr}} \delta_{\mathrm{irr}}-\sum_{i=1}^{g-1} b_{i: 1} \delta_{i: 1}-\sum_{i=0}^{g-1} b_{i: 12} \delta_{i: 12} \in \operatorname{Pic}\left(\overline{\mathcal{M}}_{g, 2}\right) .
$$

Let $\pi_{2}: \overline{\mathcal{M}}_{g, 2} \rightarrow \overline{\mathcal{M}}_{g, 1}$ be the morphism forgetting the second marked point. We claim that, the divisor $\left(\pi_{2}\right)_{*}\left(\bar{D} \cdot \Delta_{0: 12}\right)$ is trivial. Indeed, suppose this is not the case and we choose a general point $[C, p] \in\left(\pi_{2}\right)_{*}\left(\bar{D} \cdot \Delta_{0: 12}\right)$. We insert a smooth 
rational curve denoted by $\mathbf{P}^{1}$ at the point $p$ and view $p_{1}, p_{2}$ as distinct smooth points on $\mathbf{P}^{1}$. Since $\left[C \cup_{p} \mathbf{P}^{1}, p_{1}, p_{2}\right] \in \bar{D}$, there exists a limit linear series $\ell=\left\{\ell_{C}, \ell_{\mathbf{p}^{1}}\right\}$ on $C \cup_{p} \mathbf{P}^{1}$ with ramification $\alpha^{\ell}\left(p_{1}\right) \geq \bar{\alpha}^{1}$ and $\alpha^{\ell}\left(p_{2}\right) \geq \bar{\alpha}^{2}$. We write the following series of inequalities:

$$
\begin{aligned}
& -1>\rho\left(g, r, d, \bar{\alpha}^{1}, \bar{\alpha}^{2}\right) \geq \rho\left(g, r, d, \alpha^{\ell}\left(p_{1}\right), \alpha^{\ell}\left(p_{2}\right)\right) \\
& \quad \geq \rho\left(g, r, d, \alpha^{\ell}(p)\right)+\rho\left(0, r, d, \alpha^{\ell^{1}}\left(p_{1}\right), \alpha^{\ell}\left(p_{1}\right), \alpha^{\ell}\left(p_{2}\right)\right) \geq \rho\left(g, r, d, \alpha^{\ell_{C}}(p)\right),
\end{aligned}
$$

where the third inequality reflects the additivity of the Brill-Noether number that is incorporated in the definition of the limit linear series (see also [3] p. 365), whereas the last inequality follows from the Plücker formula (see also [3] Theorem 1.1). Thus $\rho\left(g, r, d, \alpha^{\ell} c(p)\right)<-1$. Theorem 0.1 guarantees that, the locus of curves $[C, p] \in \mathcal{M}_{g, 1}$ satisfying such a Brill-Noether condition is a subvariety of $\mathcal{M}_{g, 1}$ of codimension at least 2 . This leads to a contradiction since [C, $p$ ] was chosen as being a general point of a divisor on $\overline{\mathcal{M}}_{g, 1}$, therefore we conclude $\left(\pi_{2}\right)_{*}\left(\bar{D} \cdot \Delta_{0: 12}\right)=0$. At the level of classes, from the formulas

$$
\left(\pi_{2}\right)_{*}\left(\psi_{1} \cdot \delta_{0: 12}\right)=\left(\pi_{2}\right)_{*}\left(\psi_{2} \cdot \delta_{0: 12}\right)=0, \quad\left(\pi_{2}\right)_{*}\left(\delta_{i: 1} \cdot \delta_{0: 12}\right)=0 \text { for } i=1, \ldots, g-1,
$$

respectively

$$
\left(\pi_{2}\right)_{*}\left(\lambda \cdot \delta_{0: 12}\right)=\lambda, \quad\left(\pi_{2}\right)_{*}\left(\delta_{\text {irr }} \cdot \delta_{0: 12}\right)=\delta_{\text {irr }}, \quad\left(\pi_{2}\right)_{*}\left(\delta_{0: 12}^{2}\right)=-\psi_{1}, \quad\left(\pi_{2}\right)_{*}\left(\delta_{i: 12} \cdot \delta_{0: 12}\right)=\delta_{i: 1},
$$

we obtain the following identity

$$
0=\left(\pi_{2}\right)_{*}\left([\bar{D}] \cdot \delta_{0: 12}\right)=a \lambda-b_{\text {irr }} \delta_{\text {irr }}+b_{0: 12} \psi_{1}-\sum_{i=1}^{g-1} b_{i: 12} \delta_{i: 1} \in \operatorname{Pic}\left(\overline{\mathcal{M}}_{g, 1}\right),
$$

hence $a=b_{\text {irr }}=b_{i: 12}=0$, for $i=0, \ldots, g-1$.

To show that the coefficients $c_{1}$ and $c_{2}$ of $[\bar{D}]$ vanish, we note that $\operatorname{Im}\left(\varphi_{g, 2}\right) \cap \bar{D}=\emptyset$ (cf. [3] Theorem 1.1), hence $\varphi_{g, 2}^{*}([\bar{D}])=0$. We express this pull-back as an element of the Picard group Pic $\left(\overline{\mathcal{M}}_{0, g+2}\right)^{\mathfrak{S}_{g}}$, where the symmetric group $\mathfrak{S}_{g}$ acts on $\overline{\mathcal{M}}_{0, g+2}$ by permuting the first $g$ marked points of each element $\left[R, x_{1}, \ldots, x_{g}, p_{1}, p_{2}\right]$. Using for instance [1], we write the following formulas:

$$
\varphi_{g, 2}^{*}\left(\psi_{1}\right)=\psi_{p_{1}}, \quad \varphi_{g, 2}^{*}\left(\psi_{2}\right)=\psi_{p_{2}}, \quad \varphi_{g, 2}^{*}\left(\delta_{i: 1}\right)=B_{i}^{p_{1}} .
$$

Via the description of $\operatorname{Pic}\left(\overline{\mathcal{M}}_{0, g+2}\right)^{\mathfrak{S}_{g}}$ in terms of $\mathfrak{S}_{g}$-invariant boundary classes as well as the expression of the cotangent classes $\psi_{p_{1}}$ and $\psi_{p_{2}}$ given in Lemma 1.2, it is straightforward to check that the divisor classes $\psi_{p_{1}}, \psi_{p_{2}}$ and $\left\{B_{i}^{p_{1}}\right\}_{i=1}^{g-1}$ are linearly independent. In particular, from the assumption $\varphi_{g, 2}^{*}(\bar{D})=0$, we conclude that $c_{1}=c_{2}=0$. Thus we can apply Lemma 1.1, which establishes Theorem 0.2 for the case of 2 marked points.

The case $n \geq 3$ can be easily reduced to the situation discussed above:

Proof of Theorem 0.2 (the case $n \geq 3$ ). We assume, by contradiction, that the locus in moduli

$$
D:=\left\{\left[C, p_{1}, \ldots, p_{n}\right] \in \mathcal{M}_{g, n}: G_{d}^{r}\left(C,\left(p_{j}, \bar{\alpha}^{j}\right)\right) \neq \emptyset\right\}
$$

is a divisor. We shall show that the $\lambda,\left\{\psi_{j}\right\}_{i=1}^{n}$ and $\left\{\delta_{0: S}\right\}_{|S|=2}$-coefficients of $[\bar{D}]$ are zero, then use Lemma 1.1 to conclude. The inductive hypothesis coupled with [3] Theorem 1.1 ensures that $D$ enjoys two geometric properties:

(1) If $\pi_{i}: \overline{\mathcal{M}}_{g, n} \rightarrow \overline{\mathcal{M}}_{g, n-1}$ is the map forgetting the $i$-th marked point, then for each $1 \leq i \leq n$ and $j \in\{i\}^{c}$, we have that $\left(\pi_{i}\right)_{*}\left([\bar{D}] \cdot \delta_{0: i j}\right)=0$.

(2) If $\varphi_{g, n}: \overline{\mathcal{M}}_{0, g+n} \rightarrow \overline{\mathcal{M}}_{g, n}$ is the flag map, then $\varphi_{g, n}^{*}([\bar{D}])=0$.

We claim that these restrictions, together with the assumption that $D$ is effective, imply that $D=0$. Firstly, condition (1) implies that the $\lambda, \delta_{\text {irr }}, \psi_{1}, \ldots, \psi_{n}$-coefficients of $[\bar{D}]$ are equal to zero. Indeed, the $\lambda, \delta_{\text {irr }}$ and $\psi_{k}$-coefficients of the divisor classes $[\bar{D}] \in \operatorname{Pic}\left(\overline{\mathcal{M}}_{g, n}\right)$ and $\left(\pi_{i}\right)_{*}\left([\bar{D}] \cdot \delta_{0: i j}\right) \in \operatorname{Pic}\left(\overline{\mathcal{M}}_{g, n-1}\right)$ respectively, are equal for all $k \in\{i, j\}^{c}$ (this is

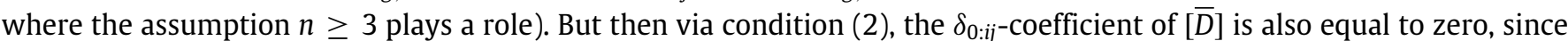
$\varphi_{g, n}^{*}\left(\delta_{0: i j}\right)=\delta_{0: p_{i} p_{j}}$. Furthermore, since all the $\psi$-coefficients in the expression $[\bar{D}]$ are zero, the coefficient of $\delta_{0: p_{i} p_{j}}$ in $\left[\varphi_{g, n}^{*}(\bar{D})\right]$ equals the $\delta_{0: i j}$-coefficient in $[\bar{D}]$, thus showing that the latter coefficient is equal to zero. We now apply Lemma 1.1 and conclude.

\section{Acknowledgment}

I would like to thank the referee for a careful reading and pertinent comments which improved the presentation.

\section{References}

[1] E. Arbarello, M. Cornalba, Calculating cohomology groups of moduli spaces of curves via algebraic geometry, Inst. Hautes Études Sci. Publ. Math. 88 (1998) 97-127. 
[2] D. Eisenbud, J. Harris, Limit linear series: Basic theory, Invent. Math. 85 (1986) 337-371.

[3] D. Eisenbud, J. Harris, The Kodaira dimension of the moduli space of curves of genus $\geq 23$, Invent. Math. 90 (1987) 359-387.

[4] D. Eisenbud, J. Harris, Irreducibility of some families of linear series with Brill-Noether number-1, Ann. Sci. Éc. Norm. Supér. 22 (1989) 33-53.

[5] G. Farkas, A. Gibney, The Mori cones of moduli spaces of curves of small genus, Trans. Amer. Math. Soc. 355 (2002) 1183-1199.

[6] R. Lehmann, Brill-Noether type theorems with a movable ramification point, arXiv:0804.4657. 